REGARDS

SUR L'ECONOMIE ALLEMAND

BULLETIN ECONOMIQUE DU CIRAC
Regards sur l'économie allemande

Bulletin économique du CIRAC

116-117| 2015

Varia

\title{
De la capacité de l'Allemagne à se réformer
}

Isabelle Bourgeois

\section{OpenEdition}

\section{Journals}

Édition électronique

URL : http://journals.openedition.org/rea/4813

DOI : 10.4000/rea.4813

ISSN : 1965-0787

Éditeur

CIRAC

Édition imprimée

Date de publication : 1 juillet 2015

Pagination : 15-28

ISSN : 1156-8992

\section{Référence électronique}

Isabelle Bourgeois, «De la capacité de l'Allemagne à se réformer », Regards sur l'économie allemande [En ligne], 116-117 | juillet 2015, mis en ligne le 01 juillet 2017, consulté le 06 mai 2019. URL : http:// journals.openedition.org/rea/4813; DOI : 10.4000/rea.4813 


\section{De la capacité de 1'Allemagne à se réformer}

\section{Isabelle Bourgeois}

En France, nous considérons non sans ambivalence notre principal voisin dans I'UE, souvent érigé en " modèle " quant aux réformes qu'il a menées depuis le tournant du millénaire - ou au contraire désigné comme " anti-modèle ». Soit nous idéalisons l'effort mené par l'Allemagne pour reconquérir sa compétitivité et préserver son modèle social tout en poursuivant une politique de consolidation budgétaire. Soit nous récusons une prospérité économique qui aurait été construite sur les ruines de l'Etat social au nom d'une rigueur budgétaire excessive. En réalité, dans les deux cas, nous commettons une erreur d'appréciation, nourrie par la difficulté à appréhender le fonctionnement des institutions comme le processus de prise de décision en Allemagne. Il est vrai que son mode d'organisation est difficile à concevoir pour nous, tant il diffère de notre système pyramidal, et tant nos visions du monde environnant et nos approches du changement sont opposées.

Quels sont ces mécanismes qui ont permis à l'Allemagne de mener des réformes structurelles ? Comment le consensus nécessaire parvient-il à émerger entre les multiples acteurs concernés et les citoyens, puis à s'installer durablement? Qu'en sera-t-il à l'avenir ? La réponse est relativement simple à formuler, même si elle recouvre un processus complexe de régulation collective : la capacité de l'Allemagne à se réformer repose sur la participation de chacun, le respect institutionnalisé de la diversité des intérêts, et une vision dynamique du changement. Le système allemand n'est certes pas facilement transposable, pas plus qu'il n'est infaillible car il comporte aussi ses blocages. Mais mieux comprendre les facteurs qui déterminent la capacité de notre partenaire à se réformer peut nous aider à œuvrer ensemble plus efficacement à la construction de l'Europe.

\section{La « germanosclérose" guérie par une succession de chocs...}

Des réformes structurelles menées par l'Allemagne, on n'a retenu pour l'essentiel en France que les réformes Hartz engagées dans le cadre de l'Agenda 2010. Cette révision en profondeur du régime de la protection chômage et de la politique active pour l'emploi est souvent présentée comme une remise en question de l'Etat social, imputable au seul chancelier Schröder (voir REA 108/ 2013). Mais on oublie alors de la considérer dans son contexte : celui d'une réforme au long cours à la fois de l'Etat providence et du modèle économique, engagée depuis le début des années 1990, portée par un long processus de prise de conscience collectif et réalisée en différentes étapes. L'Agenda 2010 n'en est que la partie la plus immédiatement visible. L'objectif déclaré de tous ces efforts était de permettre à l'économie de recouvrer sa compétitivité, c'est-à-dire d'asseoir la croissance sur des bases plus durables, afin de pérenniser à l'ère de l'économie du savoir le financement d'un Etat social dont les fondements avaient été posés au début de l'ère industrielle, sous Bismarck, et qui avait été ensuite refondé et considérablement développé durant les années du « miracle économique " (voir Lasserre, 2011). Cet objectif a été finalement atteint: les conditions réservées à l'activité permettent désormais de dégager les richesses

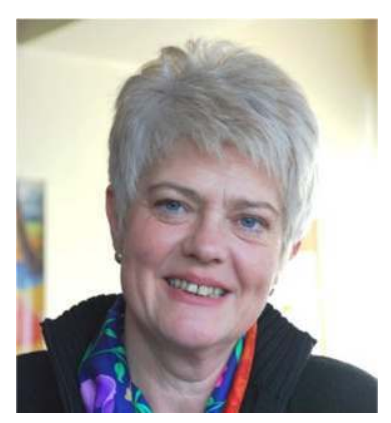

Isabelle Bourgeois, rédactrice en chef 
nécessaires pour financer le modèle social qui reste assis sur la redistribution mais dont les équilibres ont été en même temps révisés pour en assurer la soutenabilité. Ce réformisme n'est pas l'œuvre d'un seul gouvernement ni encore moins des seuls pouvoirs publics, mais de l'ensemble de la société. Peut-être est-ce là une des sources de la perplexité avec laquelle, en France, nous considérons ces réformes.

\section{“ L'Allemagne bouge » : l'Agenda 2010}

L'Agenda 2010 est un ensemble de mesures destinées à rétablir la compétitivité de l'économie pour mieux garantir la soutenabilité du modèle social. Dans la préface d'une brochure d'information publiée par l'Office de presse fédéral fin 2003 , le chancelier Schröder les présentait ainsi : "Si nous voulons que notre pays continue à assurer à ses citoyen(ne)s une vie de prospérité et de sécurité sociale, nous devons le transformer ». Huit chantiers sont engagés :

- Economie : relance des investissements dans le BTP ; mesures en faveur de la création d'entreprises (avantages fiscaux et dérogations au droit du travail, simplification de la création d'entreprises artisanales).

-Qualifications : adoption de standards nationaux d'enseignement; hausse des dépenses d'éducation, évolution des rythmes scolaires (école l'après-midi) ; obligation faite aux entreprises d'offrir des places d'apprentissage en nombre suffisant et simplification de l'habilitation à former des apprentis.

- Fiscalité : amélioration des finances communales (réforme de la taxe professionnelle) ; mesures en faveur des entreprises, dont réforme de l'imposition des plus-values de cessions; plus généralement, poursuite de l'abaissement des taux d'imposition.

- Formation/recherche : hausse des dépenses de recherche fondamentale ; «Initiative d'excellence » pour les universités. - Emploi : flexibilisation de la convention tarifaire de branche par la multiplication des accords d'entreprise ; assouplissement de la protection contre le licenciement; réformes de la politique pour l'emploi et du système de protection chômage (lois Hartz).

- Assurance maladie : abaissement des cotisations à $13 \%$ du salaire brut ; déremboursements ; fiscalisation des allocations maternité ; fin du monopole contractuel liant caisses obligatoires et médecins ; lancement d'une carte « vitale ».

- Assurance retraite : gel des pensions dans l'objectif d'abaisser le taux de cotisations à 19,5\%.

- Politique familiale : hausse des investissements dans les structures d'accueil de la petite enfance ; avantages fiscaux pour les services à domicile.

Ces chantiers se conjuguent avec les réformes et mesures des gouvernements précédents comme des acteurs de l'économie et de la société, et développent ainsi des effets cumulatifs. Ils ont lancé une dynamique favorable au changement.

Longue gestation des réformes

Quatre chocs forcent la prise de conscience

L’Unité soulève la question du financement de la protection sociale
Bien que son annonce en mars 2003 puis sa mise en œuvre ait été abrupte, et bien qu'il ait été en rupture avec les politiques précédentes qui se contentaient d'ajustements à la marge, le programme Agenda 2010 s'inscrit dans la continuité d'un processus engagé de longue date. La prise de conscience de la nécessité de procéder à des réformes en profondeur s'est en effet consolidée par poussées successives, au fil des gouvernements - de la coalition Union/FDP menée par le chancelier Helmut Kohl (1982-87) jusqu'à l'actuelle grande coalition d'Angela Merkel. Certes, en Allemagne plus qu'ailleurs, le temps politique des gouvernements est court du fait des nombreuses échéances électorales qui s'entrecroisent à l'échelon du Bund et des Länder. En revanche, le temps de la mémoire des dossiers et des enjeux à relever est lui, très long, car c'est la société civile organisée qui, par-delà les échéances politiques, conserve la maîtrise des 'dossiers techniques' et les remet régulièrement à l'ordre du jour. Entre le début de la prise de conscience d'un impératif de réforme et sa mise en œuvre effective, il peut ainsi s'écouler jusqu'à vingt ans - soit cinq législatures -, comme ce fut le cas notoire des réformes de la protection sociale.

Mais pour que cette prise de conscience progressive débouche sur des réformes de fond, il aura fallu une série de chocs. Car longtemps, l'Allemagne s'était enlisée dans l'immobilisme au point que les observateurs en étaient venus à redouter la "sclérose » d'un "modèle rhénan " confortable mais tendant à se couper du monde et surtout de l'Europe, avec tous les risques que cela faisait peser sur la compétitivité de l'ensemble de l'UE (voir Bourgeois, 2001). Quatre grands chocs principalement, au cours de la décennie 1990, sont venus amplifier la prise de conscience du besoin de réformes qui s'esquissait depuis les chocs pétroliers.

Séisme historique particulier à l'Allemagne, le choc de l'Unité (1990) a révélé au grand jour la problématique du financement d'un système de protection sociale axé sur la redistribution et reposant trop exclusivement sur le seul facteur travail. 
Du jour au lendemain, les caisses (ouest-)allemandes ont eu à verser des prestations aux assurés est-allemands ; or ceux-ci n'avaient jamais cotisé à l'ouest. La réponse arithmétique pour couvrir le déficit en termes de recettes (une forte hausse des cotisations) était exclue car elle aurait encore plus alourdi le poids des prélèvements sur les entreprises, bridant leur croissance et accroissant encore le nombre de chômeurs, déjà démultiplié par le collapsus de l'économie estallemande. Le même choc a également renforcé la prise de conscience de l'impact du vieillissement de la population : à moyen terme, les pensions de retraite ou la couverture maladie n'étaient plus finançables en l'état (voir Bourgeois, 2005).

Presque au même moment survient le choc de la crise mondiale de restructuration de l'industrie. Après le boom de l'Unité (plus de $5 \%$ de croissance en 1990 et 1991), l'économie ralentit $(2,2 \%$ en 1992$)$, avant de chuter de près de $1 \%$ en 1993. Pendant que le contexte mondial changeait, la production industrielle allemande s'était repliée sur la demande intérieure dans le fil de l'unification. Cette récession soulève pour la première fois la question de la compétitivité globale du site de production allemand (Standort Deutschland) dans une économie mondiale en mutation. Contrairement à ce qui était le cas depuis les années 1970, le débat ne porte plus simplement sur la compétitivité-prix, la fiscalité ou la réglementation, ni sur la compétitivité des entreprises. Responsables politiques et acteurs économiques s'interrogent sur les facteurs déterminants de la compétitivité du modèle économique et social allemand (voir Lallement, 1994a). Et ils s'aperçoivent que sa faiblesse dans la concurrence mondiale résidait notamment dans une réglementation trop rigide du marché du travail et de coûts de production, salariaux entre autres, disproportionnés au regard d'une productivité peu adaptée à la nouvelle donne. Il est vrai que, à la suite de la politique de rattrapage salarial menée à l'est, les salaires avaient, à l'ouest aussi, progressé sans commune mesure avec la hausse de de la productivité (voir Rürup, 2012).

Troisième choc : la genèse de l'Union économique et monétaire en 1992, puis la création de la monnaie unique en 1998 ont forcé la prise de conscience de l'impératif de consolidation budgétaire ; l'Unité avait brutalement mis fin à la politique d'assainissement engagée dans les années 1980 - il fallait bien financer par des transferts massifs la reconstruction d'une économie en ruine dans les nouveaux Länder issus de l'ex-RDA. La dette, qui avait été ramenée à $40,1 \%$ du PIB en 1989, l'année de la chute du Mur, ne cesse alors de s'accroître ; elle dépassera pour la première fois le seuil des $60 \%$ du traité de Maastricht en 1999. Depuis l'Unité et l'effort budgétaire consenti (au moins 1250 milliards $€$ ont été transférés d'ouest en est), la question de la rigueur est dès lors une priorité politique absolue en Allemagne, indépendamment des engagements européens.

Un dernier choc, externe lui aussi, contribue à maintenir la question de la compétitivité en tête de l'agenda, sous ses deux facettes - croissance et emploi : c'est la perspective de l'élargissement de l'UE en 2004. Les défis qu'il soulève sont de plusieurs ordres. Budgétaires, puisque dans ce cadre, les fonds structurels européens allaient être redirigés pour l'essentiel vers les nouveaux Etats membres est-européens, et que l'Allemagne allait voir se réduire ceux auxquels étaient jusqu'alors éligibles les nouveaux Länder de l'est. En termes de politique de croissance, puisqu'il fallait créer les conditions pour que l'Allemagne en général, ses Länder de l'est en particulier, soient en mesure de puiser dans leurs propres forces pour développer et consolider leur dynamique. Ce défi reposait donc la question de la compétitivité économique et appelait à améliorer les conditions-cadre pour permettre aux entreprises de prospérer, d'investir, d'innover et d'embaucher - et cela, dans une situation de concurrence démultipliée au sein même de l'UE comme sur le marché domestique. Enfin, le défi se pose également en termes de politique sociale : comment prendre en compte la nouvelle mobilité au sein de l'UE élargie et la concurrence
La crise mondiale de l'industrie pose celle de la compétitivité

L'UEM, celle de la soutenabilité des finances publiques

L'élargissement de l'UE repose celle de la compétitivité et de l'emploi 
d'une main-d'œuvre à coût salarial moindre tout en luttant contre le chômage à l'intérieur du pays ?

\section{... et d'appels à réforme de plus en plus pressants}

Ces chocs - et même en un sens les implications budgétaires, économiques et sociales de l'Unité - ont longtemps été considérés comme conjoncturels et appelé des réponses ponctuelles se résumant la plupart du temps à des modifications à la marge des politiques antérieures. Même les enjeux fonciers qu'on commençait à entrevoir ont suscité une réaction proche de la procrastination. Rappelons que les défis s'enchaînaient au point de s'entremêler, et qu'il était souvent plus simple ou plus opportun de se contenter de 'réformettes' plutôt que de tenter de démêler l'écheveau accumulé en s'attaquant à des réformes de fond. Tirer sur un fil aurait nécessité de dénouer aussi tous les autres. Des travaux d'Hercule peu compatibles avec l'enchaînement des échéances électorales...

Pourtant, dans cette situation d'immobilisme chronique, entretenue par un néocorporatisme inhérent au "capitalisme rhénan » et favorable au statu quo social et sociétal (voir Uterwedde, 2005), à plusieurs reprises, pouvoirs publics ou experts indépendants avaient depuis les années 1980 établi des constats circonstanciés et lancé des appels à réforme dont l'un des plus mémorables reste le "discours sur le sursaut " prononcé en avril 1997 par le président Roman Herzog (voir infra). S'il n'a pas été suivi d'effet lui non plus, la répétition et l'accumulation des analyses et propositions de diagnostic, formulées par des instances comme l'OCDE ou, plus percutantes en matière de politique intérieure, celles du Conseil des Sages, auront néanmoins fini par créer une rémanence du besoin de réformes structurelles qui a été finalement favorable au changement.

1982 :

première remise en question des acquis sociaux

En réaction à la crise économique et à la hausse subite du chômage déclenchées par le premier choc pétrolier (1973) et le début des mutations structurelles de l'industrie, le chancelier Helmut Schmidt (SPD) mène une politique de relance néo-keynésienne par la consommation et de traitement social du chômage, financée par le recours à l'endettement. En 1982, l'Allemagne est en récession. Le ministre des Finances du chancelier Schmidt, Otto Graf Lambsdorff (FDP), s'oppose alors au recours à la dette et à la revalorisation des prestations sociales, plaidant au contraire pour une révision des acquis sociaux dont le périmètre avait été considérablement étendu au fur et à mesure que renaissait la prospérité. Dans son ébauche d'une politique dédiée à la croissance et l'emploi, il explique: "Se cramponner à des prestations publiques qui ne sont plus finançables dorénavant a pour conséquence inévitable d'aggraver les problèmes de croissance et d'emploi et de plonger encore plus l'Etat dans un engrenage de répartition qui, en pénalisant toujours plus la performance et la prévoyance individuelles, entretient le développement d'une logique de revendication et d'une mentalité d'assisté " (Lambsdorff, 1982). Cette divergence provoque la rupture de la coalition SPD/FDP en 1982 et la composition d'un nouveau gouvernement CDU/CSU-FDP mené par Helmut Kohl (CDU). Quelque vingt ans plus tard, le chancelier Gerhard Schröder (SPD) rappellera dans son discours de politique générale du 14-032003 que "la structure de nos systèmes de protection sociale est restée pratiquement inchangée depuis cinquante ans ", avant de poursuivre un peu plus loin : " la vocation foncière de l'Etat social ne se résume pas à la simple répartition de moyens financiers".

1993 : le tournant de la "Préservation de l'avenir du site Allemagne "...
La dégradation de la compétitivité constatée lors de la crise mondiale de l'industrie, le faible impact des transferts massifs sur la dynamique de transition des nouveaux Länder ou l'emploi amènent des diagnostics très pessimistes. L'économiste américain R. Dornbusch va même jusqu'à intituler " The End of the German Miracle" un article publié en juin 1993 dans le Journal of 
Economic Literature (voir Lallement, 1994a). Peu à peu, la conscience se dégage que cette crise est structurelle, et qu'elle appelle de ce fait une réponse concertée, de toutes les parties concernées : entreprises, partenaires sociaux et pouvoirs publics (voir Lallement, 1994b). En septembre 1993, le gouvernement fédéral publie un rapport sur la «Préservation de l'avenir du site Allemagne » («Zukunftssicherung des Standortes Deutschland»). II propose la thérapie suivante: "L'avenir des performances de notre site dépend foncièrement des valeurs et attitudes mentales qui déterminent le climat de la société allemande. Nous ... ne devons pas nous adonner à l'illusion que nous pouvons nous reposer sur nos acquis... Le changement nécessaire ne peut pas être le seul fait de l'Etat ou de la sphère politique. La préservation du site Allemagne concerne tout un chacun: le Bund, les Länder et les communes aussi bien que les partis politiques, les partenaires sociaux, les fédérations professionnelles et les Eglises, ou encore les écoles et les institutions culturelles, médias inclus » (Bundesregierung, 1993).

En septembre 1993 est votée une loi sur la « sauvegarde du site Allemagne » (Standortsicherungsgesetz). Mais, comme le souligne le Conseil des Sages dans son rapport annuel publié deux mois plus tard, "l'essentiel du programme du gouvernement fédéral est formulé en des termes très généraux. Les priorités n'y sont pas vraiment définies. C'est à la fois décevant et préoccupant " (Sachverständigenrat, 1993). Certes, le "Programme d'action pour plus de croissance et d'emplois» («Aktionsprogramm für mehr Wachstum und Beschäftigung », Bundesregierung, 1994) d'une soixantaine de mesures présenté dans la foulée par la coalition Union/FDP manque de congruence, malgré les allègements fiscaux en faveur des entreprises. Mais son objectif premier est d'entretenir une dynamique collective grâce à une politique de l'offre permettant aux acteurs économiques de reprendre confiance.

L'orientation générale de cette politique de l'offre remporte en tout cas l'adhésion de nombreux sociaux-démocrates, dont Karl Schiller, qui fut le ministre de l'Economie du chancelier Kurt Georg Kiesinger (CDU) dans le premier gouvernement de grande coalition Union/SPD (1966-69). Il est l'un des maîtres d'œuvre de la Loi pour la promotion de la stabilité et de la croissance (Gesetz zur Förderung der Stabilität und des Wachstums der Wirtschaft) adoptée en 1967 et qui constitue jusqu'à aujourd'hui le droit canon de toute politique de croissance allemande. Elle fait obligation à l'Etat de travailler à concilier quatre objectifs pour réaliser l'équilibre global de l'économie : stabilité des prix, taux d'emploi élevé, croissance et équilibre des échanges extérieurs. Elle requiert un «pilotage global» («Globalsteuerung») conjuguant politiques fiscale, monétaire et salariale, qui requiert une «action concertée » ( konzertierte Aktion ») associant Bund, Länder, communes, banque centrale, représentants des entreprises, mouvement syndical et milieux scientifiques. Le gouvernement fédéral se contente de donner des impulsions au plan macro-économique (il fixe des conditions-cadre : Ordnungspolitik), laissant par ailleurs agir librement les lois du marché et les acteurs privés. Karl Schiller résumait cette approche par la formule : " marché autant que possible, Etat autant que nécessaire », en référence à celle contenue dans le Programme de Bad Godesberg adopté par le SPD en 1959 («concurrence autant que possible, planification autant que nécessaire »). L'Etat n'a à intervenir qu'en cas de dysfonctionnements avérés. Or, au fur et à mesure que se renforçaient les blocages entre partenaires sociaux (« néo-corporatisme »), la planification d'Etat avait fini par prendre le dessus. Et en 1994, constatant lui aussi une dérive néo-keynésienne de l'approche initiale de l'action concertée, Karl Schiller résume par cette phrase le défi que tente de relever le gouvernement de coalition CDU/ FDP du chancelier Kohl : "II nous faut beaucoup plus une politique de croissance qu'une politique de répartition » (cité par $\mathrm{R}$. Lallement, 1994b).

Pourtant, malgré nombre de réformes sectorielles, le statu quo qui caractérise l'Allemagne durant ces années entretient l'immobilisme. Après un bref rebond en 1994, la croissance reste en deçà de son potentiel jusqu'au tournant du

\section{... et rééquilibrage de la politique} du «pilotage global »

1997 : Roman Herzog, «discours sur le sursaut" 
1997/98 : Le Conseil des Sages redéfinit la politique de l'offre

«La croissance n'est pas réalisée par l'Etat »

2000 : Stratégie de Lisbonne millénaire. Certains acquis sociaux ont été réduits, mais aucun des freins diagnostiqués n'a été réellement levé (voir Bourgeois/Lasserre, 2009). En 1997, l'Allemagne est installée dans ce que certains observateurs étrangers nomment la «germanosclérose ». La construction européenne avance à pas de géant, la mondialisation s'accélère, mais l'Allemagne reste comme prise de léthargie par rapport aux défis que cela implique. Cette situation incite le président de la République Roman Herzog (CDU) à lui consacrer le discours qu'il tient le 26 avril 1997 à l'Hôtel Adlon à Berlin et qui s'est inscrit dans les mémoires sous le surnom de « discours sur le sursaut». En voici l'extrait le plus célèbre : "Quel est le mal qui ronge notre pays? N'ayons pas peur des mots pour désigner la crise: la perte du dynamisme économique, une société sclérosée, une incroyable dépression mentale... les gens se sentent dépassés par les innombrables changements nécessaires simultanément... le monde est en train de changer, il n'attend pas l'Allemagne. Mais il n'est pas trop tard. Un sursaut doit traverser l'Allemagne » (discours «Aufbruch ins 21. Jahrhundert », 1997, surnommé «Ruckrede»).

Dans son rapport annuel intitulé «Croissance, emploi, Union monétaire orientations pour l'avenir » («Wachstum, Beschäftigung, Währungsunion Orientierungen für die Zukunft ») qu'il présente à la fin 1997, le Conseil des Sages place l'impératif des réformes dans son contexte global. "Alors que l'environnement mondial subit de profondes mutations et dans la perspective de l'impact qu'auront l'union monétaire comme l'élargissement de l'UE à l'est, il importe d'améliorer les conditions permettant que l'initiative privée débouche sur des activités marchandes afin qu'un plus grand nombre de personnes trouve un emploi et afin que soit préservée la prospérité ». Et le Conseil, gardien de l'orthodoxie ordolibérale du «libéralisme organisé », formule des recommandations reposant toutes sur une approche renouvelée de la notion de «politique de l'offre».

Sa nouvelle définition tient compte de l'évolutivité du contexte : une politique de l'offre consiste à " créer un environnement fiable et stable » pour l'activité. Cela implique une "politique économique orientée sur le long terme » (§ 293) et menée en coopération par trois types d'acteurs : l'Etat avec sa politique budgétaire et fiscale, la Bundesbank avec sa politique monétaire, et les partenaires sociaux avec «leur autonomie et leur responsabilité en matière de politique salariale ». Cette définition est toujours actuelle, il suffit de remplacer «Bundesbank» par «Banque centrale européenne». Le fonctionnement de cette coopération repose sur une logique contractuelle établie sur la confiance mutuelle. Ce qui «lui donne vie, c'est que les prestations générées dans un domaine sont honorées par d'autres acteurs et que, en retour, les prestations préalables ne peuvent voir le jour que si ceux qui les génèrent peuvent se reposer en toute confiance sur l'attente de les voir honorées" (§ 294). Autrement dit : "la croissance n'est pas réalisée par l'Etat ; elle est au contraire la résultante de ces innombrables efforts humains » (§ 295). Enfin, le Conseil des Sages souligne qu'une telle politique de l'offre est d'autant plus incontournable dans l'UE que «les politiques économiques nationales voient leurs effets largement limités par le degré croissant d'interdépendance des relations économiques ». Quant à la globalisation, elle " aggrave les problèmes des pays qui ne parviennent pas à aménager les conditions réservées à l'offre de telle manière que la main-d'œuvre disponible soit occupée sur le marché de la production. De mauvaises conditions réservées à l'offre sont le résultat des manquements et négligences domestiques » (§ 306).

Malgré un effort sensible de mise en conformité de la politique nationale avec l'intégration croissante du marché communautaire et la prise en compte du facteur démographique dans le calcul des retraites, les innombrables recommandations de réforme restent largement lettre morte. Le premier gouvernement SPD/Verts élu à l'automne $1998\left(1^{\mathrm{er}}\right.$ mandat du chancelier Schröder $)$ mène à l'inverse une politique néokeynésienne déclarée. Or la dette continue à se creu- 
ser, le chômage poursuit inexorablement sa progression, la compétitivité de l'économie reste structurellement faible. La dérive des coûts de l'Etat providence, qui accapare plus de $40 \%$ du PIB, n'est plus soutenable. Ce dernier constat vaut pareillement pour les autres Etats de l'UE qui, lors du Sommet européen de Lisbonne en 2000, s'engagent dès lors à engager des réformes structurelles pour dynamiser leur compétitivité et, partant, celle de l'UE (voir Bourgeois/Gabel, 2007). La Stratégie de Lisbonne qui en est issue, aussi baptisée "Croissance et emploi ", vise à rompre avec la spirale infernale d'un Etat social qui accapare une grande partie des richesses au détriment de l'investissement, de l'innovation, de l'emploi et de la soutenabilité des finances publiques comme du lien social. Cette Stratégie, dont l'objectif suprême est de préserver le modèle social grâce à une compétitivité recouvrée, restera pourtant lettre morte, "en grande partie faute d'engagement et de volonté politique » de la part des Etats membres, comme le résume le rapport de suivi européen publié en 2004 par le Groupe de haut niveau présidé par Wim Kok (Rapport Kok, 2004).

Seule l'Allemagne mettra en œuvre cette Stratégie, du moins en partie. II est vrai qu'elle y est tenue par l'art. 23 de la Loi fondamentale : "Pour l'édification d'une Europe unie, la République fédérale d'Allemagne concourt au développement de l'Union européenne". II est le "pivot constitutionnel de 'louverture de l'Etat' (offene Staatlichkeit) allemand à son intégration dans I'UE » (Müller-Graff, 2012). Dès lors, dans son discours de politique générale du 14 mars 2003 où il présente l'Agenda 2010, G. Schröder assume ouvertement cet engagement européen: "Pour respecter la responsabilité de l'Allemagne en Europe et pour l'Europe, nous devons être prêts au changement en interne ". Et le chancelier, dont la coalition avait été reconduite de justesse à l'automne 2002, souligne: "Nous devons améliorer les conditions-cadre pour générer croissance et emplois ».

Après que le premier gouvernement Schröder avait opéré un recentrage de sa politique au tournant du millénaire, et que le deuxième s'était contenté de présenter, en arrivant au pouvoir quelques mois plus tôt, un catalogue de bonnes intentions, le chancelier renoue ouvertement avec l'orthodoxie. L'Agenda 2010 suit largement les recommandations formulées à l'automne 2002 par le Conseil des Sages dans son rapport intitulé : « 20 points pour la croissance et l'emploi » (“Zwanzig Punkte für Beschäftigung und Wachstum»). Le Conseil avait fixé des priorités : flexibiliser le marché de l'emploi pour faciliter l'embauche et ainsi élargir l'assiette des cotisations sociales tout en réduisant le volume des transferts sociaux. La marge de manœuvre reconquise dans le domaine des budgets publics et sociaux devant permettre à terme de réduire le poids des prélèvements. "Nous ne pouvons répartir que les richesses que nous avons au préalable créées », rappelait G. Schröder pour justifier l'appel au "courage d'engager le changement " qui était le fil conducteur de son discours du 14 mars 2003.

\section{Les facteurs systémiques de l'aptitude au changement de l'Allemagne}

Les réformes structurelles allemandes sont donc le fruit d'une lente maturation, succession de pas en avant et en arrière, d'échappatoires et de brusques dénouements. Elles n'ont jamais non plus été décidées ex nihilo par le politique seul. Bien au contraire, le plus souvent, les pouvoirs publics n'ont apporté que la pierre finale à un édifice déjà en grande partie construit par les partenaires sociaux et les acteurs économiques. L'appel à réforme que lançait le Conseil des Sages dans son rapport 2002/03 est limpide à cet égard. En conclusion de son catalogue, il rappelait: "La mise en œuvre et l'efficacité d'un programme pour l'emploi et la croissance ne requièrent pas seulement une volonté politique forte, mais tout autant des structures décisionnelles efficientes". Autrement
2003 : l'Allemagne assume sa responsabilité européenne

L'Agenda 2010 s'inspire des recommandations du Conseil des Sages 
Les réformes sont le fruit de « compromis à l'allemande »

dit : un système décisionnel basé sur la coopération et associant les citoyens, détenteurs premiers du pouvoir dans la démocratie allemande. La transparence qui est le préalable à la formation de la volonté collective est assurée par les médias.

Les réformes de fond sont ainsi le fruit de patients et savants ajustements. Une fois décidées, elles sont d'autant plus facilement mises en œuvre qu'elles sont collectivement assumées : chaque partie est informée en permanence des enjeux comme de l'évolution des négociations en cours, et peut manifester son opposition via les représentants de ses intérêts et infléchir les réflexions jusqu'à ce que soit trouvé un accord permettant de concilier tous les intérêts en jeu. C'est cet équilibre construit ensemble et respectueux des intérêts légitimes respectifs qu'on dénomme généralement un «compromis à l'allemande »: un compromis gagnant-gagnant. C'est parce qu'il n'y a pas de perdant que chaque partie peut s'identifier avec l'accord conclu, que celui-ci est consensuel, c'est-àdire vécu positivement, et dès lors respecté. Ce mode de fonctionnement réserve une place établie à la critique, considérée comme constructive car elle apporte des perspectives nouvelles au débat et donc l'enrichit. Une fois les mesures décidées, le suivi critique assure l'accompagnement de leur mise en œuvre et permet d'apporter les correctifs éventuellement nécessaires. Par définition, toute réforme allemande est donc un processus à la fois long et en perpétuel développement.

Cette place éminente accordée au compromis ne relève pas génériquement de la culture. Elle est bien plutôt une nécessité ou, plus précisément, un mécanisme structurel lié à l'organisation même des institutions et de la société de la République fédérale d'Allemagne. Cette «culture du consensus » est en réalité systémique : les Allemands n'ont pas d'autre choix positif que de trouver des compromis, alors que le conflit, qui conduit au blocage, est vécu comme un échec.

\section{Allemagne. Des réformes perpétuelles et collectives}

Les principales réformes (ponctuelles et structurelles) engagées en Allemagne depuis les années 1990

Par les entreprises

A partir de la crise mondiale de l'industrie, les entreprises industrielles se restructurent et rationalisent leur production. Cet effort de compétitivité est porté par les salariés, dont la représentation élue dans l'entreprise (Betriebsrat) est co-responsable dans tous les domaines relevant de l'organisation de la production.

\section{Par les partenaires sociaux}

Syndicats et fédérations patronales de branche œuvrent à flexibiliser la réglementation du travail. Au début des années 1990, dans l'est de l'Allemagne, les entreprises en difficulté bénéficient de clauses d'ouverture leur permettant de ne pas appliquer la hausse salariale conclue à l'échelon de la branche. Cette pratique dérogatoire est ensuite étendue, dans certaines conditions, à l'ensemble de l'Allemagne.

A partir de 2001 sont signés plusieurs accords d'entreprise dans des grands groupes (Bosch, Siemens, Daimler), portant sur la modulation et la hausse du temps de travail hebdomadaire sans compensation salariale. En 2004 est développé chez Volkswagen un modèle de flexibilité interne qui se généralisera ensuite sous les formes les plus diverses.

En 2004, IG Metall et Gesamtmetall signent l'Accord de Pforzheim : la clause d'ouverture s'applique désormais aussi aux entreprises prospères pour leur permettre d'accroître leur compétitivité (voir Schrœder, 2005).

La fonction publique est modernisée, la durée du temps de travail portée à 42 heures.

\section{Par les pouvoirs publics}

Fiscalité : allègements fiscaux en faveur des entreprises (1993) ; relèvement du taux de TVA de $15 \%$ à $16 \%$ (1998) ; adoption en 1999 d'une réforme en trois étapes (1999, 2000, 2003) visant à alléger l'IR et l'impôt sur les bénéfices ; création de l'écotaxe pour développer les énergies renouvelables et abaisser les cotisations retraite (1999); baisse de la contribution de l'Etat à l'assurance retraite et gel des pensions (2000); la Loi de réduction fiscale abaisse le poids des prélèvements (IR et impôt sur les bénéfices) et exonère de l'impôt les plus-values sur cessions de valeurs mobilières (2000); hausse du taux de la TVA de $16 \%$ à $19 \%$ dont un tiers est affecté à l'abaissement des cotisations chômage et deux tiers à la réduction du déficit budgétaire (2006) ; réforme de la fiscalité des entreprises $(2007) \ldots$

Assurance maladie : instauration d'un budget global pour les soins et médicaments, augmentation de la participation des assurés (1993) ; réduction des indemnités et compléments de salaires en cas de maladie (1995); hausse de la participation (1997/2002) ; baisse la part patronale des cotisations, modulation des tickets modulateurs (2003) ; création d'un Fonds santé (2007, Gesundheitsfonds : mutualisation de l'ensemble des cotisations salariales et d'une taxe versée par les contribuables et répartition entre les caisses selon un montant forfaitaire)...

Assurance retraite : la Loi de réforme de l'assurance retraite (1997) prend en compte le facteur démographique (rapport actifs/ inactifs) dans le calcul des retraites ; création de l'écotaxe comme contribution au financement du régime des retraites légales (1998) ; lancement de la « retraite Riester » : complément par capitalisation (épargne retraite subventionnée ; 2001) ; prise en 
ration du vieillissement de la population et de la hausse de l'espérance de vie, allongement progressif de l'âge légal de la retraite à 67 ans (2007) (voir Bontout et al., 2013)...

n d'une assurance dépendance (1995), réformée en $2014 .$.

u travail et protection chômage : assouplissement du régime du travail intérimaire (1995, 1997), du travail à temps 1996), de la protection contre le licenciement (1996), des emplois dits « minimes » $(1999,2003)$; modification en 1997 i sur la promotion du travail de 1969 : l'objectif de stabilisation d'un haut niveau d'activité est remplacé par celui de la on du chômage et de l'efficience accrue du placement des demandeurs d'emploi ; lois Hartz (2002/05) : réforme de fédérale pour l'emploi, baisse de la durée de versement des allocations dans le régime d'assurance chômage, création uveau régime d'assistance forfaitaire, soumis à conditions de revenus, pour ceux qui ne peuvent prétendre aux allocaômage, ainsi que leurs proches (voir REA 108/2013 ; Hazouard et al., 2013)...

isme financier : intégration des nouveaux Länder dans la péréquation horizontale entre Länder (Länderfinanzaus1995) ; réforme du fédéralisme politique : clarification de la répartition des compétences entre Bund et Länder (2006) ; in d'un pacte de stabilité interne à la RFA (2009; voir REA 102/2011 et 115/2014)...

Du fait de l'organisation fédérale de l'Allemagne, sa première caractéristique est la polyarchie. Les décisions politiques sont prises par 17 gouvernements, dont le gouvernement fédéral. Celui-ci ne peut prendre (presque) aucune décision d'envergure sans l'aval des 16 Länder. Car le fédéralisme allemand est coopératif et solidaire (voir REA 102/2011) : les pouvoirs sont partagés entre la Fédération et les Länder, c'est-à-dire à la fois répartis entre ces deux échelons et exercés conjointement dans le respect des intérêts particuliers (Länder) comme de l'intérêt supérieur (RFA). Les Länder étant des Etats fédérés largement souverains, ils co-décident à l'échelon supérieur de la politique fédérale via leur représentation: le Bundesrat. Cette co-décision est destinée à garantir la cohésion de cet ensemble d'acteurs autonomes et indépendants, exerçant chacun ses responsabilités à son niveau, qu'est l'Allemagne; c'est là l'aspect le mieux connu en France du principe de subsidiarité. Les majorités des gouvernements régionaux et donc les politiques menées dans chaque Land étant très diverses, les Länder sont tenus à une attitude propice à l'unité de la fédération ("bundesfreundliches Verhalten"), qui découle de l'art. 20, al. 1 de la Loi fondamentale : la conclusion de compromis respectueux des intérêts de l'ensemble de la RFA.

Or, au fil de l'évolution des rouages du fédéralisme, et au fur et à mesure que la progression de l'intégration européenne amenait l'Allemagne à transférer un nombre croissant de compétences à l'échelon communautaire, cette forme de parlementarisme à l'origine a pris de plus en plus l'allure d'une méthode intergouvernementale (le Bundesrat est composé de chefs de gouvernement), avec pour effet de réduire progressivement les pouvoirs du Bundestag, parallèlement amenuisés par le renforcement des processus décisionnels à l'échelon communautaire. C'est dans cette évolution qu'il faut voir le principal motif de la Cour de Karlsruhe lorsqu'elle cherche, notamment dans son arrêt rendu à propos du MES, à renforcer les pouvoirs du Bundestag (voir Müller-Graff, 2011 ; 2012). Cette « déparlementarisation », connue et critiquée depuis longtemps (voir $\mathrm{Pa}$ pier, 2003), figure à l'arrière-plan des deux réformes du fédéralisme en 2006 et 2009, comme de celles à venir. Les critiques visent toutes à rétablir ou renforcer les mécanismes de co-décision au niveau des pouvoirs institutionnels.

Les intérêts en jeu étant complexes, les blocages sont nombreux. Les échéances électorales du Bund et de chacun des Länder étant distinctes les unes des autres, la composition du Bundesrat varie fréquemment, et il peut se transformer en chambre d'opposition pour le gouvernement fédéral. C'est ainsi par exemple que la grande réforme fiscale adoptée en 2002 par le premier gouvernement Schröder avait connu une genèse de près de dix ans. Le projet avait été engagé en 1995 par le gouvernement Kohl, vidé de sa substance en 1996 dans le cadre des négociations Bund-Länder, reformulé puis enterré en 1997 du fait de l'obstruction du Bundesrat alors à majorité SPD, avant de renaître presque à l'identique du texte d'origine et d'être finalement adopté en 2000 sous la nouvelle coalition SPD/Verts (voir Bourgeois, REA 54/2001). Entretenue par l'appel à réforme incessant dans l'espace public, et profitant de 
Un système politique axé sur la coopération l'alternance politique, la raison économique a fini par l'emporter sur les considérations partisanes.

Le mode de scrutin mixte, à la fois majoritaire et proportionnel, des élections au Bundestag, de même que la barre des $5 \%$ des voix que doit franchir tout parti pour y entrer, forcent l'élection de gouvernements de coalition. Dans l'histoire de la RFA, aucun parti n'a jamais formé seul le gouvernement fédéral. Une « grande coalition » rassemblant CDU/CSU et SPD, c'est-à-dire le centre droit et le centre-gauche, est parfaitement naturelle, même si elle est rare : l'actuelle Grande coalition n'est que la troisième. Dans les Länder aussi, les partis politiques doivent en permanence travailler ensemble dans les constellations les plus diverses. Tous les partis sont donc rompus à la pratique des responsabilités et à celle de la négociation. Certes, ce fédéralisme coopératif inter-partis peut se traduire par des marchandages épiques (les réformes du fédéralisme financier en sont un bon exemple; voir REA 115/2014), mais au final, il débouche toujours sur un accord. Et si celui-ci ne se révèle pas satisfaisant à l'usage, il peut être renégocié. C'est là une des raisons qui explique par exemple pourquoi l'Union CDU/CSU a adhéré au projet de salaire minimum légal porté par le SPD alors même qu'elle y était farouchement opposée. Mais cet étroit entrelacement institutionnalisé des intérêts politiques peut aussi nourrir l'immobilisme; ce fut longtemps le cas après l'Unité dans le domaine des réformes structurelles, avant l'Agenda 2010.

II n'y a donc pas de clivage gauche/droite tel que nous le connaissons en France : tous les partis (sauf le nouveau parti populiste et eurosceptique AfD ; et dans une certaine mesure le néo-marxiste Die Linke) se situent au centre de l'échiquier politique, ne se distinguant que par des accentuations tempérées sur les enjeux centraux. Tous sont des partis de rassemblement (Volksparteien) se définissant "par un souci constant de fédérer - et, ce faisant, de dépasser - les différentes positions idéologiques et les groupes d'intérêts présents dans la société allemande » (Uterwedde, 2007). En effet, les partis " concourent à la formation de la volonté politique du peuple » ; c'est ainsi que la Loi fondamentale définit leur mission première (art. 21, al. 1). Participant de "l'ordre constitutionnel libéral et démocratique" (freiheitlich-demokratische Grundordnung) de la RFA, ils partagent la doctrine de l'économie sociale de marché et l'intégration de l'Allemagne dans l'UE.

Partenariat social : l'équilibre des intérêts

II y a également partage des compétences et donc coopération institutionnalisée entre les pouvoirs publics et la société civile organisée. Dans le domaine du travail, les partenaires sociaux représentent ainsi les intérêts du salariat et du patronat. L'exécutif n'a aucune compétence constitutionnelle pour fixer les salaires ou les conditions de travail ; syndicats et fédérations patronales sont seuls compétents en la matière, chacun au niveau de sa branche. Le salaire minimum légal adopté par l'actuelle Grande coalition en 2013 ne constitue pas foncièrement une entorse à ce principe, l'autonomie tarifaire des partenaires sociaux étant dans l'ensemble respectée grâce au recours à quelques aménagements légaux qui en garantissent la normalité. Or les règles des négociations sur les conventions collectives qui s'ouvrent tous les deux ans en moyenne dans chaque branche ont pour finalité de permettre de trouver un terrain d'entente malgré des intérêts parfois exacerbés, débouchant sur une convention établie par contrat et dont la date d'expiration et le mode de révision font partie intégrante. Ce système d'équilibre des intérêts vise à garantir la paix sociale et la prévisibilité des conditions réservées à l'activité. Même les mouvements catégoriels qui se multiplient actuellement dans le secteur des anciens services publics récemment «dérégulés 》 ne peuvent se soustraire à cette règle du compromis organisé.

Par ailleurs, à l'échelon de chaque gouvernement, les intérêts institutionnalisés du patronat et du salariat sont statutairement associés à toute décision législative les concernant. Aucune décision politique ne peut être prise par 
l'exécutif sans l'aval de la "triade décisionnelle »: Confédération syndicale DGB, Fédération des employeurs (BDA), Fédération de l'industrie (BDI). Cela n'interdit toutefois pas à un gouvernement de céder à la tentation de se décharger sur les partenaires sociaux d'une partie de ses fonctions. Ce fut le cas par exemple des «Pactes pour l'emploi » institués à la fin des années 1990 par les gouvernements Kohl, puis Schröder, pour définir une politique de lutte contre le chômage. Ils furent un échec, les partenaires sociaux estimant qu'ils avaient apporté de leur côté, dans leur champ de compétences, une contribution majeure à la modernisation de la production en flexibilisant les conditions d'emploi et de travail par la négociation et qu'il revenait à l'exécutif de prendre ses responsabilités dans son domaine d'intervention et d'apporter sa contribution complémentaire en modernisant le cadre légal : les normes de droit du régime de la protection chômage ou les règles de licenciement. La réponse du gouvernement Schröder II fut d'instituer une Commission chargée d'élaborer des propositions pour une modernisation du marché du travail et une réorganisation complète des institutions en charge des régimes d'assurance chômage et d'assistance: la Commission Hartz. Elle était composée de personnalités politiques, d'experts, d'universitaires et, bien sûr de représentants des syndicats et des fédérations patronales; mais la responsabilité finale incombait au seul législateur.

Le recours croissant à des commissions a pour finalité de créer un consensus et d'entretenir une adhésion la plus large possible dans l'espace public. Car elles sont toujours composées de représentants des organisations de la société. Ces commissions ad hoc peuvent se voir confier la mission de travailler sur des dossiers 'techniques', comme c'est le cas du Comité d'experts institué en août 2014 pour élaborer une stratégie d'investissement. Leur institution peut aussi viser à contourner les réticences des partenaires sociaux, notamment du mouvement syndical, comme ce fut le cas de la Commission Hartz. Or cette 'commissionnite' est vertement critiquée en Allemagne, le politique refusant ainsi d'assumer ses responsabilités et de porter les conséquences de son action. II est vrai que l'institution d'une commission n'a souvent qu'un seul but : celui de faire donner par la société civile sa bénédiction, sinon son 'absolution', à une option politique fortement controversée. Le dernier exemple en date se situe certes hors du champ social mais concerne des choix de société d'une haute importance: la sortie accélérée du nucléaire décidée en 2011. La Commission chargée d'entériner ce choix aux enjeux essentiellement économiques était la... Commission d'éthique.

Ces commissions-là sont éphémères et font partie intégrante de la sphère étatique. II n'en va pas de même des innombrables institutions vouées à l'expertise scientifique ou à l'évaluation des politiques publiques, et qui jouent un rôle primor-dial en Allemagne, où le gouvernement fédéral est tributaire de l'avis d'experts extérieurs, issus de la société civile, pour asseoir la légitimité de son action. Ces institutions sont toujours indépendantes, installées hors des cercles universitaires ou administratifs, ce qui est une garantie du pluralisme de l'analyse (voir Gabel/Lasserre, 2006). Elles peuvent émaner des partenaires sociaux (Fondation Hans Böckler pour le mouvement syndical, Institut der deutschen Wirtschaft pour le patronat et l'industrie), être financées partiellement sur des fonds publics comme les grands instituts de conjoncture (ifo, DIW, IfW, RWI, IWH, HWWA ou ZEW), être proches du monde universitaire (Instituts Max Planck, SWP ou WZB), être issues d'initiatives privées (Alfred-HerrhausenGesellschaft, Institut für Demoskopie Allensbach) ou encore émaner des partis politiques (Fondations Konrad Adenauer, Friedrich Ebert, Friedrich Naumann). Cette grande diversité, reflet des forces présentes dans la société, assure un dialogue permanent et pluraliste des approches dans le débat public tout en contribuant à éclairer les citoyens.

Pluralisme de l'expertise et du conseil politique... 
... et contribution à la formation de la volonté politique

Symbiose entre société civile organisée et pouvoirs publics
Parmi ces institutions, qui poursuivent toutes une mission d'intérêt général, un rôle particulier revient au Conseil des Sages, le Sachverständigenrat zur Begutachtung der gesamtwirtschaftlichen Entwicklung (SVR). Statutairement indépendant du pouvoir politique, composé de cinq professeurs d'économie d'obédiences diverses, ce Conseil avait été institué par voie législative en 1967 et s'était vu confier une mission d'intérêt public : celle "d'évaluer l'évolution économique et d'éclairer le jugement de toutes les instances responsables en matière de politique économique ainsi que de l'opinion publique ». II publie tous les ans, en novembre, un rapport annuel comportant critiques et recommandations, et le soumet au gouvernement fédéral qui dispose jusqu'à la mi-janvier pour prendre position sur ses avis dans le rapport présentant son programme de politique économique pour l'année en cours. Le gouvernement fédéral doit donc tenir compte de l'évaluation par cette haute autorité externe, d'une réputation scientifique incontestable. Car, quelle que soit l'obédience du gouvernement fédéral, elle a la fonction de gardien de la doctrine ordolibérale : elle « s'attache imperturbablement à maintenir ou à faire prévaloir par des mesures et des règles du jeu appropriées (Ordnungspolitik) un cadre général favorable à l'activité économique et à la compétitivité globales 》(Gabel/Lasserre, 2006). Rapport annuel et réponse du gouvernement sont largement diffusés dans l'espace public et minutieusement débattus, voire vertement critiqués dans les médias. Cela aussi contribue à éclairer les citoyens sur les enjeux politiques et leur permet de comprendre le pourquoi des réformes. De même, cela contribue à consolider l'identification des Allemands avec les fondements de leur « modèle économique et social » tel que l'a progressivement constitutionnalisé la Cour de Karlsruhe (voir REA 52-53/2001) : l'ordolibéralisme et l'économie sociale de marché.

Aucun gouvernement ne peut mener son action sans tenir effectivement compte des principaux intérêts exprimés par la société civile organisée. Tous les intérêts particuliers des diverses composantes de la société sont légitimement représentés par le biais d'associations ayant pignon sur rue et ont une place institutionnalisée dans le débat public. Leur rôle d'articulation va bien audelà du simple lobbying : grâce à un dialogue permanent à la fois au sein de la société et avec les pouvoirs publics, il contribue en effet à la définition collective de l'intérêt général, faisant de ce dernier une sorte de compromis consensuel entre la multitude des perspectives en présence. La définition de ce consensus national est donc par nature évolutive et s'adapte en permanence aux mutations de la société comme à celles des grands enjeux économiques et sociaux. En Allemagne, "ce n'est donc pas le primat du politique sur les groupes d'intérêt et le contrat social qui domine, mais au contraire une approche de symbiose entre pouvoirs publics et société civile " (Uterwedde, 2005).

Ce ne sont pas les pouvoirs publics qui sont détenteurs du pouvoir dans la démocratie allemande, mais bien le peuple à travers ses multiples représentations: "Tout pouvoir émane du peuple. Le peuple l'exerce au moyen d'élections et de votations et par des organes spéciaux investis des pouvoirs législatif, exécutif et judiciaire » (art. 20 al. 2 de la Loi fondamentale). Via son droit de vote, le citoyen leur délègue mandat pour exercer ces pouvoirs en son nom. Le droit de vote n'est en effet «pas un acte formel de légitimation de la puissance publique », il vise d'abord "à garantir l'exercice effectif de la souveraineté du peuple » (Müller-Graff, 2011). C'est là l'expression première du principe de subsidiarité, relayée ensuite par de multiples représentations civiles organisées.

En effet, le pouvoir du peuple s'exerce également via la fonction de contrôle qu'ont ses représentants en tant qu'acteurs de la société ou de l'économie. Ces innombrables organisations sont une autre institution fondamentale de la démocratie allemande: elles sont l'autre forme de représentation de la volonté du peuple, aux côtés du Bundestag (ou d'un Landtag). Alors que le parlement se 
compose de représentants élus par les citoyens et œuvre à préserver l'intérêt général, les organisations de la société civile (Verbände) permettent l'articulation institutionnalisée des intérêts particuliers. Ces deux types de représentations sont complémentaires. Et c'est seulement quand il est possible de trouver un équilibre entre intérêts particuliers et général qu'un consensus porteur peut se dégager. "Les Verbände font partie intrinsèque du capital social d'une société ; ils mettent à sa disposition une infrastructure de relations bien réglées et établies au sein de chacune des composantes de la société et entre celles-ci, grâce auxquelles il est possible de mettre fin aux conflits et de chercher et trouver les intérêts communs » (Streeck, 1999b).

Dans le domaine économique, trois catégories de Verbände se distinguent: d'une part les fédérations des acteurs économiques à adhésion obligatoire ( $\mathrm{CCl}$, chambres des métiers) ou fortement recommandée (fédérations sectorielles de l'industrie, par exemple) et dont le rôle premier est de faire valoir les intérêts de leurs membres comme de les tenir informés en permanence de l'évolution économique ou réglementaire. D'autre part, les fédérations à fonction opérative que sont les partenaires sociaux dans chaque branche où ils influent directement sur le cadre réservé aux activités: c'est le niveau du dialogue social de branche. Enfin, celles qui ont une fonction politique au niveau des institutions étatiques dans la mesure où elles sont associées aux prises de décision : ce sont la Confédération syndicale DGB et la Fédération patronale $\mathrm{BDA}$, ainsi que les fédérations de l'industrie (BDI), des $\mathrm{CCl}$ (DIHK) et des chambres des métiers (ZDH).

BDA, BDI, DIHK et ZDH constituent ce qu'il est convenu d'appeler les « fédérations au sommet » (Spitzenverbände). Sans leur consentement, aucune décision politique ne peut être prise. Leur opposition incite souvent les gouvernements à changer de cap. Ainsi en 1999, lorsqu'elles ont contraint le premier gouvernement Schröder à abandonner son cap néo-keynésien d'inspiration quelque peu dirigiste et ont forcé à la démission le ministre fédéral des Finances Oskar Lafontaine qui, dans la phase de naissance de I'UEM, avait 'sommé' la BCE de baisser ses taux. Les mêmes, par leurs appels à réforme incessants, ont contribué à déterminer le gouvernement Schröder à lancer l'Agenda 2010. Elles mettent en garde les gouvernements contre les tentations, en période conjoncturelle faste, de ralentir l'effort de réformes et de redistribuer les richesses. Ainsi, par exemple, BDI, BDA, DIHK et ZDH avaient publié le 17 octobre 2007 un appel pressant au gouvernement fédéral ("Poursuivre la politique de réforme ! ") à ne pas diluer les réformes Hartz, expliquant : "L'Allemagne ne peut pas se permettre de se livrer à une politique populiste du retour en arrière, alors que le reste du monde affronte le changement nécessaire. $\mathrm{La}$ concurrence mondiale ne s'arrête pas ».

Ce genre de prise de position, de même que les appels répétés de la Bundesbank, des grands instituts de conjoncture ou du Conseil des Sages (son rapport annuel 2013/14 s'intitulait : "Contre une politique économique du retour en ar rière ") sont de puissantes cordes de rappel. Toutes ces positions, de même que les voix opposantes, sont amplement relatées et commentées par les médias où elles donnent lieu à débat permanent. Les médias assument une fonction "au service» du processus démocratique, comme ne cesse de le souligner la Cour de Karlsruhe depuis 1958 (" arrêt Lüth », BVerfGe 12, 198), et jouent pleinement leur rôle d'information, d'articulation, de critique et de contrôle. Leur indépendance vis-à-vis du pouvoir politique découle de l'art. 5 de la Loi fondamentale qui tire les enseignements de deux dictatures $\left({ }^{\mathrm{II}}{ }^{\mathrm{e}}\right.$ Reich et RDA) en garantissant non seulement la liberté d'expression individuelle, mais aussi la liberté institutionnelle des médias, avec son corollaire très rare en démocratie : le droit à l'information. Les citoyens sont donc toujours informés des projets politiques et des prises de position respectives dans l'espace public. Ils en comprennent d'autant plus aisément les enjeux qu'ils sont au fait des lois économiques et des impératifs des entreprises, connaissances acquises et

Transparence démocratique et citoyens éclairés 
transmises notamment grâce à l'apprentissage dans le système dual. Ce « quatrième pouvoir » polycentrique et pluraliste est le facteur clé de la cohésion de cette " société apprenante » qu'est l'Allemagne - le mécanisme par excellence de cette "culture " de la concertation que requiert un système institutionnel multi-acteurs.

LE « MODELE ALLEMAND » EST PAR NATURE EVOLUTIF, contrairement à ce qu'on est porté à croire en France. Seuls les grands principes fondateurs de l'ordre étatique sont invariants ; la forme concrète que leur donne le législateur varie selon les impératifs internes et externes. "Chaque pays doit se réformer en permanence car le contexte ne cesse d'évoluer et de soulever de nouveaux défis ", explique par exemple Roman Herzog, qui fut président de la Cour de Karlsruhe, puis président de la RFA (Konvent für Deutschland, 2014). La capacité de l'Allemagne à s'adapter et à mener des réformes structurelles repose sur une vision positive et partagée du changement : celle d'une économie et d'une société ouvertes - sur le monde et sur l'Europe -, mais aussi ouvertes à toutes les voix dans le respect de leur diversité. C'est là le corollaire d'un système institutionnel polyarchique en quête perpétuelle d'équilibre, dont le système médiatique qui assure un flux d'information permanent et omnidirectionnel est le facteur clé de cohésion.

Le mécanisme clé de l'adaptabilité du « modèle allemand " peut dès lors se résumer à un grand principe organisateur : la confiance. Et son corollaire : cette logique du contrat sur laquelle repose son mode de fonctionnement institutionnel. Construite en interne sur le respect des multiples intérêts et la pratique des choix collectifs complexes et multi-acteurs, l'Allemagne exerce et assume tout naturellement son rôle dans une Union européenne elle aussi " unie dans la diversité " et évolutive. L'Allemagne n'est pas «modèle ». Son fonctionnement est simplement en cohérence avec la dynamique de la globalisation et de la construction européenne.

\section{Indications bibliographiques}

- BDI, BDA, DIHK, ZDH, « Aufruf der deutschen Wirtschaft. Reformpolitik fortsetzen! » (http://arbeit geber.de/www/arbeitgeber.nsf/id/771520E238D04C79C12573770020B774?open\&ccm=999660)

- Bontout O., HazouARD S., LasserRe R., Zaidman C. (dir.)., Les réformes de la protection sociale en Allemagne. Etat des lieux et dialogue avec des experts français, CIRAC/DREES, 2013

- Bourgeols I. (dir.), Le modèle social allemand en mutation, CIRAC, Cergy-Pontoise, 2005

- Bourgeols I. (dir.), Allemagne 2001. Regards sur une économie en mutation, CIRAC, Cergy-Pontoise, 2001

- BouRgeols I., «Les réformes Hartz, remise en cause de l'Etat social ? ", « Années Schröder : les 'recentrages' successifs de la politique économique et sociale ", "Réforme fiscale allemande : la lente construction des équilibres d'Helmut Kohl à Gerhard Schröder », Regards sur l'économie allemande, $n^{\circ} 108$, printemps 2013, $n^{\circ} 73$, octobre 2005, n 54, décembre 2001

- Bourgeols I., Gabel M., "Stratégie de Lisbonne. Perspectives franco-allemandes ", Les notes de l'Ifri, $\mathrm{n}^{\circ}$ 59, 2007

- Bourgeois I., LAsserRe R., La République fédérale d'Allemagne. Chronique politique, économique et sociale. 1949-2009, CIRAC, Cergy-Pontoise, 2009

- BundesRegierung, Bericht der Bundesregierung über den Stand der Umsetzung der Maßnahmen zur Zukunftssicherung des Standortes Deutschland und des Aktionsprogramms für mehr Wachstum und Beschäftigung, BT-Drucksache 12/6907, 1994 (http://dip21.bundestag.de/dip21/btd/12/069/1206907.pdf)

- BundeSREgIERUNG, Bericht der Bundesregierung zur Zukunftssicherung des Standortes Deutschland, BT-Drucksache 12/5620, 1993 (http://dipbt.bundestag.de/doc/btd/12/056/1205620.pdf)

- FLACHeneCKer F., «Politische Reformfähigkeit in der Eurozone », Deutsche Bank Research, Research Briefing Europäische Integration, 03-02-2012 (www.dbresearch.de)

- Gabel M., LASSerRe R., « L'expertise économique et le conseil politique à l'épreuve du changement », Regards sur l'économie allemande, $n^{\circ}$ 78, octobre 2006

- "Gesetz zur Verbesserung der steuerlichen Bedingungen und zur Sicherung des Wirtschaftsstandorts Deutschlands im europäischen Binnenmarkt (Standortsicherungsgesetz - StandOG) ", 13-09-1993 (www.gesetze-im-internet.de/standog/BJNR156900993.html)

- « Gesetz zur Förderung des Stabilität und des Wachstums der Wirtschaft (StabG) », 08-06-1967 (www.gesetze-im-internet.de/bundesrecht/stabg/gesamt.pdf)

- Hazouard S., LasserRe R., Uterwedde H. (dir.), L'aide au retour à l'emploi. Politiques françaises, allemandes et internationales, CIRAC, Cergy-Pontoise, 2013 
- Herzog R., «Aufbruch ins 21. Jahrhundert », discours prononcé à Berlin le 26-04-1997 (www.bund Herzog/Reden/1997/04/19970426_Rede.html) espraesident.de/SharedDocs/Reden/DE/Roman-

- Kammann U., “Compagnons de voyage. Les médias et l'Unité allemande », in Bourgeols I. (dir.), Allemagne, les chemins de l'unité. Reconstruction d'une identité en douze tableaux, CIRAC, Cergy-Pontoise, 2011

- Konvent für Deutschland, Reform der Reformfähigkeit, Berlin, mai 2014 (www.konvent-fuerdeutschland.de)

- LaLlement R. (1994a), « La compétitivité de l'Allemagne comme espace économique. Le made in Germany en question. I. Diagnostic », et (1994b) « La compétitivité de l'Allemagne comme espace économique. Le made in Germany en question. II. Thérapie », Regards sur l'économie allemande, $\mathrm{n}^{\circ} 15$, mars 1994, et $\mathrm{n}^{\circ} 16$, mai 1994

- Lambsdorf O., Konzept für eine Politik zur Überwindung der Wachstumsschwäche und zur Bekämpfung der Arbeitslosigkeit, Bonn, 1982

- LASSERRE R., « La formation professionnelle en Allemagne. Dynamiques socio-économiques et capacités d'adaptation du système dual », Regards sur l'économie allemande, n 113, été 2014

- LASSERRE R., " La réforme de l'Etat social en Allemagne », in Bourgeols I. (dir.), Allemagne, les chemins de l'Unité. Reconstruction d'une identité en douze tableaux, CIRAC, Cergy-Pontoise, 2011

- LASSERre R., «Le redressement économique de l'Allemagne », Politique étrangère, n 4 , hiver 2007

- MüLleR-Graff P.-C., «L'arrêt de Karlsruhe à propos du MES », et « L'arrêt de Karlsruhe sur les aides budgétaires dans la zone Euro ", Regards sur l'économie allemande, n 106, automne 2012, et $n^{\circ} 102$, automne 2011

- PAPIER H.-J., « Steuerungs- und Reformfähigkeit des Staates », Conférence tenue le 10 avril 2003 à la Stiftung für Ordnungspolitik, Fribourg-en-Brisgau

- Relever le défi. La stratégie de Lisbonne pour la croissance et l'emploi, Rapport du Groupe de haut niveau présidé par Wim Kok, novembre 2004

- RüRup B., « L'Agenda 2010, un modèle pour la France ? », Regards sur l'économie allemande, $\mathrm{n}^{\circ} 107$, hiver 2012

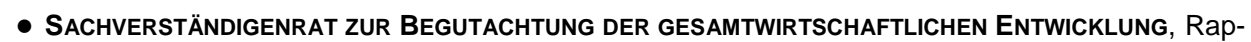
ports annuels, consultables sur www.sachverstaendigenrat-wirtschaft.de. En particulier :

1992/93 : «Für Wachstumsorientierung - Gegen lähmenden Verteilungsstreit »; 1993/94 : «Zeit zum Handeln - Antriebskräfte stärken »; 1995/96 : «Im Standortwettbewerb ; 1997/98: «Wachstum, Beschäftigung, Währungsunion - Orientierungen für die Zukunft ; 1999/2000: «Wirtschaftspolitik unter Reformdruck»; 2002/03: «Zwanzig Punkte für Beschäftigung und Wachstum » ; 2005/06 : «Die Chance nutzen - Reformen mutig voranbringen » 2009/10: «Die Zukunft nicht aufs Spiel setzen »; 2011/12 : «Verantwortung für Europa wahrnehmen »; 2012/13 : "Stabile Architektur für Europa - Handlungsbedarf im Inland ; 2013/14: "Gegen eine rückwärtsgewandte Wirtschaftspolitik »; 2014/15 : «Mehr Vertrauen in Marktprozesse »

- Schröder G., «'Mut zum Frieden und zur Veränderung », discours de politique générale prononcé le 14 mars 2003 au Bundestag, Bundestag, Plenarprotokoll 15/32 (http://dip21.bundestag.de/ dip21/ btp/15/15032.pdf)

- SChroeder W., «'Le modèle syndical allemand n'existe plus' », in BouRgeoıs I. (dir.), Le modèle social allemand en mutation, CIRAC, Cergy-Pontoise, 2005

- SPD, Godesberger Programm. Grundsatzprogramm der SPD, 13/15-11-1959, www.spd.de/linkabl eblob/1816/data/godesberger_programm.pdf

- StreECK W., Korporatismus in Deutschland. Zwischen Nationalstaat und Europäischer Union, Francfort-Main/New York, 1999 (1999a)

- StReecK W., « Verbände als soziales Kapital : Von Nutzen und Nutzung des Korporatismus in einer Gesellschaft im Wandel ", contribution à un symposium organisé à l'occasion du $50^{\mathrm{e}}$ anniversaire de la Fédération BDI, Cologne, 11-12 mars 1999 (1999b)

- UTERWEDde H., «Les programmes de la CDU et du SPD : convergences de fond », Regards sur l'économie allemande, $\mathrm{n}^{\circ} 84$, décembre 2007

- UTERWEdde H., «'Capitalisme rhénan': défis d'adaptation et compétitivité virtuelle », in BOURGEOIS I. (dir.), Le modèle social allemand en mutation, Editions du CIRAC, 2005. 